\title{
Evidence for Specialized Rhythm-Generating Mechanisms in the Adult Mammalian Spinal Cord
}

\author{
Alain Frigon and Jean-Pierre Gossard \\ Groupe de Recherche sur le Système Nerveux Central des Fonds de la Recherche en Santé du Québec, Département de Physiologie, Université de Montréal, \\ Montréal, Québec H3C3J7, Canada
}

Locomotion and scratch are characterized by alternation of flexion and extension phases within one hindlimb, which are mediated by rhythm-generating circuitry within the spinal cord. By definition, the rhythm generator controls cycle period, phase durations, and phase transitions. The aim was to determine whether rhythm-generating mechanisms for locomotion and scratch are similar in adult decerebrate cats. The regulation of cycle period during fictive scratching was evaluated, as were the effects of specific sensory inputs on phase durations and transitions during spontaneous fictive locomotion and pinna-evoked fictive scratching. Results show that cycle period during fictive scratching varied predominantly with flexion phase duration, contrary to spontaneous fictive locomotion, where cycle period varied with extension phase duration. Ankle dorsiflexion greatly increased extension phase duration and cycle period during fictive locomotion but did not alter cycle period during scratching. Moreover, stimulating the plantaris (ankle extensor muscle) nerve during flexion reset the locomotor rhythm to extension but not the scratch rhythm. Stimulating the plantaris nerve during extension prolonged the extension phase and cycle period during fictive locomotion but not during fictive scratching. Stimulating the sartorius nerve (hip flexor muscle) during early flexion reduced the flexion phase and cycle period during fictive locomotion, but considerably prolonged the flexion phase and cycle period during fictive scratching. These data indicate that cycle period, phase durations, and phase transitions are not regulated similarly during fictive locomotion and scratching, with or without sensory inputs, providing evidence for specialized rhythm-generating mechanisms within the adult mammalian spinal cord.

\section{Introduction}

Central pattern generators (CPGs) within the spinal cord produce rhythmic hindlimb movements (for review, see Delcomyn, 1980; Grillner, 1981; Kiehn, 2006; McCrea and Rybak, 2008). The functional organization of spinal CPGs is however unclear, particularly in mammals. In some CPG models, rhythm generation is separated from pattern formation to account for changes in neuronal activity that do not influence the "clock function" of the rhythm (McCrea and Rybak, 2008). By definition, the rhythm generator controls cycle period (i.e., the time between successive bursts of activity recorded in a given nerve or muscle) and phase durations, while the pattern formation module coordinates and distributes inputs to specific motor pools. According to such a scheme, different rhythmic motor behaviors could be mediated by a single rhythm generator (Berkinblit et al., 1978a,b; Gelfand et al., 1988; Perreault et al., 1999). However, there could be different configurations of the rhythm-generating circuitry, with specialized mechanisms for different tasks.

Received Jan. 27, 2010; revised March 31, 2010; accepted April 12, 2010.

The present work was supported by the Canadian Institutes of Health Research (J.-P.G.) and by the Christopher and Dana Reeve Foundation (A.F.). All studies were conducted in the Département de Physiologie at the Université de Montréal. We thank France Lebel for technical assistance and Jennifer Sirois for help with some of the experiments.

Correspondence should be addressed to Dr. Alain Frigon, Northwestern University, Department of Physiology, Feinberg School of Medicine, 303 E. Chicago Avenue, Chicago IL, 60611. E-mail: frigon.alain@gmail.com.

DOI:10.1523/JNEUROSCI.0450-10.2010

Copyright $\odot 2010$ the authors $\quad 0270-6474 / 10 / 307061-11 \$ 15.00 / 0$
There is ample evidence that different rhythmic motor behaviors are produced by shared and specialized circuitry within the spinal cord (Stein, 2005; Berkowitz, 2007, 2008; Li et al., 2007; Liao and Fetcho, 2008; McLean et al., 2008; Frigon, 2009). If one rhythm generator (i.e., one configuration of the rhythmgenerating circuitry) mediates multiple rhythmic motor behaviors, there should be similar control mechanisms from one task to another. Alternatively, if different rhythmic movements have specialized control mechanisms it would provide evidence for distinct configurations of the rhythm-generating circuitry. Consequently, systematically investigating similarities and differences from one task to another could provide important clues regarding the functional organization and reorganization of CPGs.

Recently, we showed that cycle period was regulated by primarily varying the extension phase during spontaneous fictive locomotion in decerebrate cats and during pharmacologically evoked fictive locomotion in acute or chronic cats with complete spinal transection (Frigon and Gossard, 2009). The first aim of the present study was to evaluate the regulation of cycle period during fictive scratch in the adult cat. The second aim was to determine whether specific sensory inputs influence phase durations and transitions similarly during fictive locomotion and scratching (i.e., rhythm resetting). The ability of sensory inputs to reset the rhythm is generally taken as evidence that signals are directly accessing the rhythm-generating circuitry (Conway et al., 1987; Gossard et al., 1994; Kriellaars et al., 1994; Hultborn et al., 1998; McCrea, 1998; Pearson et al., 1998; Schomburg et al., 
1998). In particular, inputs from hip and ankle muscle afferents exert potent effects on the rhythm-generating circuitry during fictive locomotion in adult cats (Conway et al., 1987; Guertin et al., 1995; Perreault et al., 1995; Schomburg et al., 1998; Stecina et al., 2005; Frigon et al., 2010), but the effects of these inputs during fictive scratch have received little attention (Baev et al., 1991; Perreault et al., 1999). A difference in the regulation of cycle period and in the ability to reset the cycle during fictive locomotion and scratch would provide evidence that both rhythms have specialized control mechanisms.

\section{Materials and Methods}

General procedure and ethical information. All procedures were in accordance with the Guide for Care and Use of Experimental Animals (Canada) and approved by the Ethics Committee of the Universite de Montréal. All animals were obtained from a designated breeding establishment for scientific research. Before the experiments, animals were housed and fed within designated areas, which are monitored daily by veterinarians and trained personnel. Some of the data presented in the current study are derived from experiments performed within the last 15 years for answering other scientific questions, which is part of our effort to maximize the scientific output of every animal experiment. A total of 32 cats were used to compile the present dataset.

Before surgery, cats were injected with an analgesic (Anafen $2 \mathrm{mg} / \mathrm{kg}$, s.c.) and premedicated (Atravet $0.1 \mathrm{mg} / \mathrm{kg}$, glycopyrrolate $0.01 \mathrm{mg} / \mathrm{kg}$, ketamine $5-10 \mathrm{mg} / \mathrm{kg}, \mathrm{i} . \mathrm{m}$.). Cats were then anesthetized with a mask using a mixture of oxygen $(\sim 50 \%)$, nitrous oxide $(\sim 50 \%)$ and isoflurane (2-4\%, Abbott Laboratories). Once the animal was deeply anesthetized (10-15 $\mathrm{min})$, a tracheotomy was performed and cats were intubated to provide the anesthesia. The right common carotid artery was cannulated to monitor blood pressure and the right jugular and cephalic veins were cannulated for fluid administration. The level of anesthesia was confirmed by monitoring blood pressure, applying pressure to the paw to detect limb withdrawal, and by verifying the size and reactivity of the pupils.

Following a craniotomy, the cortex was removed and all tissue rostral to the colliculi and mammillary bodies was removed (i.e., a precollicular/ premammillary decerebration). At this point, animals are considered to have complete lack of sentience. Anesthesia was discontinued and animals were paralyzed with an injection $(1 \mathrm{mg} / \mathrm{kg})$ of pancuronium bromide (Sandoz Canada Inc.) through the right jugular or cephalic veins. Paralysis is required to remove movement-related sensory feedback to study the centrally generated motor patterns (i.e., "fictive" locomotion or scratching) and the effects of specific sensory inputs. Immediately after this injection, the animals were artificially ventilated for the duration of the experiment. An identical injection of pancuronium bromide was administered every $45 \mathrm{~min}$ until the end of the experiment. A lethal injection of pentobarbital anesthetic was administered at the end of the experiment through the right jugular or cephalic veins.

Recordings and stimulation. A spontaneous fictive locomotor rhythm is frequently observed $(>50 \%)$ after a precollicular/premammillary decerebration. Manually stimulating the neck region and pinna or short trains of electrical stimuli to the sciatic nerve were sometimes used to initiate episodes of fictive locomotion, which could last for several minutes, and hours in some cases. To evoke fictive scratch a laminectomy was made at C1-C2 and after removing the dura, a small cotton ball soaked in D-tubocurarine ( $0.1 \%$; Sigma) was applied topically (Feldberg and Fleischhauer, 1960). Episodes of fictive scratching occurred spontaneously or were induced by manual stimulation of the left pinna (Deliagina et al., 1975; Côté and Gossard, 2003). The position of the cat within the stereotaxic frame was the same in all experiments, which is important because the joint positions of the recorded hindlimb influences cycle and phase durations during fictive locomotion (Pearson and Rossignol, 1991) and scratching (Berkinblit et al., 1978b).

To monitor fictive scratching or locomotion, the electroneurography (ENG) of selected nerves of the left hindlimb was performed by dissecting and mounting the following nerves on bipolar silver-chloride hook electrodes: posterior biceps-semitendinosus (PBSt), semimembranosus- anterior biceps (SmAB), lateral gastrocnemius-soleus (LGS), medial gastrocnemius (MG), plantaris (Pl), flexor hallucis longus/flexor digitorum longus (FDHL), tibialis anterior (TA), extensor digitorum longus (EDL), and sciatic nerve (uncut). The superficial peroneal (SP) nerve was also mounted uncut. In some experiments, the sartorius (Sart) nerve was placed in a cuff electrode. In a few experiments some peripheral nerves, such as MG and LGS, were left uncut to investigate the effects of a slight dorsiflexion of the ankle during episodes of fictive scratch or locomotion. Electroneurography was bandpass filtered $(10 \mathrm{~Hz}-10 \mathrm{kHz})$ and amplified. Episodes of fictive scratch and locomotion were digitized on-line with interactive custom-made software (Spinal Cord Research Center, University of Manitoba, Winnipeg, MB, Canada) or off-line from recordings on videotape (15 channels; Vetter 4000A). Data were analyzed using the same custom-made software.

After a laminectomy exposing spinal segments L5-S1, the animals were transferred to a stereotaxic frame and skin flaps surrounding the spinal cord and the hindlimb nerves were used to construct paraffin oil pools. The cord dorsum potential (CDP) was recorded with a silver chloride ball electrode and stimulation intensity was expressed as multiples of the threshold ( $\mathrm{T}$ ) for the most excitable fibers in the nerve determined by the first negative deflection of the CDP. During some episodes of fictive scratching or locomotion, Pl, LGS, or Sart nerves were stimulated using isolated units (World Precision Instruments) driven by a Master 8 stimulator (A.M.P.I.) with trains of 25,50 , or 100 pulses at $200 \mathrm{~Hz}$ at $1.8 \mathrm{~T}, 2 \mathrm{~T}$, or $5 \mathrm{~T}$.

Data analysis. Overall, 130 episodes of fictive scratching $(n=62)$ and locomotion $(n=68)$ were analyzed in 32 cats. These consisted of 28 episodes of scratching in 15 cats, 11 episodes of scratching before and during dorsiflexion in 4 cats, 23 episodes of fictive scratching with nerve stimulation in 5 cats, 13 episodes of fictive locomotion before and during dorsiflexion in 4 cats, and 55 episodes of fictive locomotion with nerve stimulation in 14 cats. Some of the data with nerve stimulation during fictive locomotion was recently presented in a different format for a study concentrating on the effects of stimulating hip afferents during spontaneous fictive locomotion (Frigon et al., 2010).

The duration of the flexion and extension phase was inferred by measuring the duration of the flexor and extensor bursts, respectively. Burst duration was calculated as the time between burst onset and termination from the raw ENG waveforms, whereas cycle period was defined as the time between successive flexor $\left(\mathrm{CP}_{\mathrm{FLEX}}\right)$ and extensor $\left(\mathrm{CP}_{\mathrm{EXT}}\right)$ burst onsets in a given nerve (Fig. $1 B, C$ ). During fictive scratching, the extension phase is very brief and does not cover most of the interval between TA bursts. As a result, the interval between TA bursts was also calculated to determine whether this interval varied with cycle period. The flexor burst interval was calculated from TA burst offset to TA burst onset (Fig. $1 C)$. Regression lines were fitted to scatter plots of phase duration relative to its corresponding cycle period (e.g., flexion phase duration plotted against $\left.\mathrm{CP}_{\mathrm{FLEX}}\right)$. Coefficients of linear regression $(r$, the slope of the relationship) were calculated using SigmaPlot 10.0 (Systat Software). The ENG with the best signal-to-noise ratio was used to measure phases and cycle periods. The ENG from LGS, SmAB, MG, or Pl, which have approximately the same onsets and offsets during fictive locomotion or scratch (Deliagina et al., 1981; Grillner, 1981; Rossignol, 1996), were used for extension whereas ENG from TA was used for flexion.

Statistical analysis. All statistical tests were done using statistical software (SPSS 15.0). A simple linear regression was performed between phase duration (i.e., dependent variable) and cycle period (i.e., independent variable) and an $F$ test was used to determine whether the regression was significant. In some instances, a multiple linear regression was performed to determine significant differences between the slopes of the flexion $\left(r_{\text {FLEX }}\right)$, extension $\left(r_{\text {EXT }}\right)$, or flexion-interval $\left(r_{\text {FLEX-INT }}\right)$ phase duration/cycle period regression lines. The dependent variable (phase duration) was compared against three independent variables [cycle period, phase (flexion or extension), and cycle period $\times$ phase]. The regression coefficient for cycle period $\times$ phase determines whether $r_{\mathrm{FLEX}}, r_{\mathrm{EXT}}$, and $r_{\text {FLEX-INT }}$, significantly differ during an episode. This analysis was performed because cycle period can covary (or remain invariant) with the flexion and extension phases concomitantly.

To determine whether locomotor or scratch episodes were extensoror flexor-dominated 3 criteria were used: (1) a slope $>0.5$ or more neg- 
A

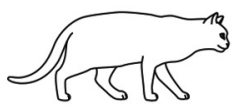

Fictive locomotion

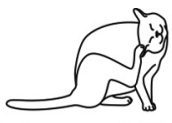

Fictive scratching
Pinna stim

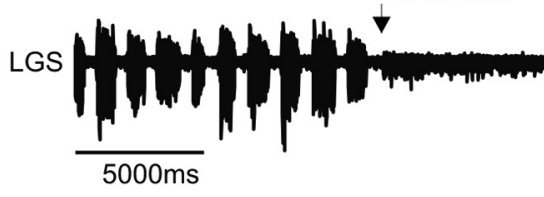

TA

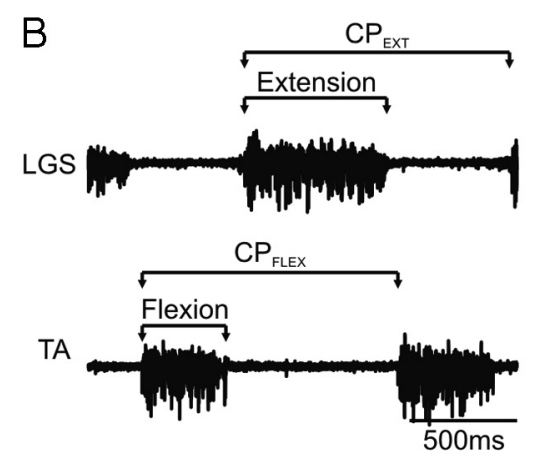

$\mathrm{D}$

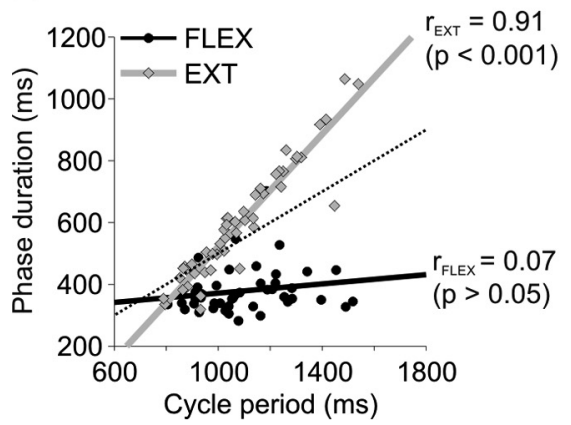

C

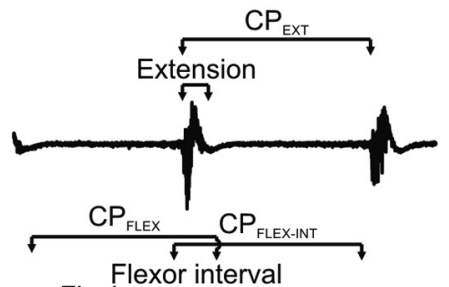

Flexor interval

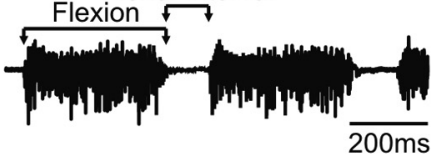

E

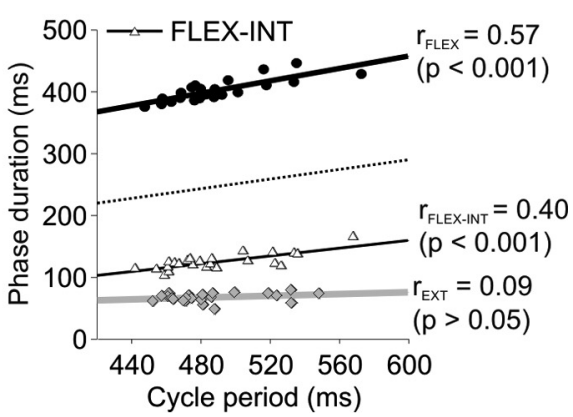

Figure 1. Regulation of cycle period during a switch from spontaneous fictive locomotion to fictive scratching in the same cat. $A$, ENG from extensor (LGS) and flexor (TA) nerves during spontaneous fictive locomotion followed by pinna stimulation and fictive scratching in one cat. $\boldsymbol{B}, \boldsymbol{C}$, Measurements during fictive locomotion $(\boldsymbol{B})$ and scratching $(\boldsymbol{C}) \cdot \boldsymbol{D}, \boldsymbol{E}$, Scatter plots and regression lines for flexion (black circles), extension (gray diamonds), and flexion-interval (white triangles) phase durations expressed relative to the corresponding cycle period during spontaneous fictive locomotion (D) and pinna-evoked fictive scratching $(\boldsymbol{E})$ for the episode illustrated in $\boldsymbol{A}$. The dotted line has a slope of 0.5 and passes through the origin and is used as a reference line.

used to determine significant differences before and during ankle dorsiflexion for the same parameters during fictive locomotion and scratching. The flexion-interval phase was only calculated during fictive scratching. Paired $t$ tests were also performed to determine whether nerve stimulation significantly altered phase durations and cycle period during fictive locomotion and scratching in 4 subdivisions of the cycle, termed "epochs of stimulation." These epochs consisted of early (EF), mid(MF), and late (LF) flexion, and of the extension (EXT) or flexion-interval (FLEX-INT) phases during fictive locomotion and scratching, respectively. The flexion-interval phase during fictive scratching is analogous to the extension phase of fictive locomotion. Six epochs were previously used during spontaneous fictive locomotion, dividing the extension phase into early, mid-, and late components (Frigon et al., 2010), but because the extension phase is so short during fictive scratching all stimulation falling during the extension (i.e., locomotion) or flexion-interval (i.e., scratching) phase were grouped together to make direct comparisons between the two rhythms. With stimulation during $\mathrm{EF}, \mathrm{MF}$, or LF the duration of the flexion phase that fell within (F1) and followed (F2) the stimulation were measured and compared with control flexion phases. With stimulation during EXT or FLEX-INT, the durations of the extension or flexion-interval phases were measured and compared with control extension or flexion-interval phases. F2 was also measured with stimulation during EXT or FLEX-INT, and compared with control flexion phases. The cycle period from successive TA burst onsets during $\left(\mathrm{CP}_{\text {stim }}\right)$ and after $\left(\mathrm{CP}_{\text {after }}\right)$ the stimulation were measured and expressed relative to control cycles (i.e., the 2-3 cycles that preceded the stimulation). Significance for all statistical tests is $p<0.05$. Numerical values in the text are the mean $\pm S D$, whereas values in the histograms are the mean \pm SE.

\section{Results}

Regulation of cycle period during

fictive scratching

During normal walking, cycle period is regulated by changing the duration of the extension phase (i.e., stance), while the flexion phase (i.e., swing) remains relatively invariant (Goslow et al., 1973; Grillner et al.,

ative than $-0.5,(2)$ a significant regression, and (3) a significant difference between $r_{\text {FLEX }}, r_{\text {EXT }}$, and $r_{\text {FLEX-INT }}$ if two or more of the slopes were $>0.5$. For example, an extensor-dominated episode has a $r_{\mathrm{EXT}}>0.5$ (or more negative than -0.5 ), the regression is significant, and $r_{\text {FLEX }}$ and $r_{\text {FLEX-INT }}$ do not exceed 0.5. An episode that covaries has two slopes or more that are $>0.5$ (or more negative than -0.5 ) and two or more slopes that are not significantly different from each other. An episode that does not vary has all slopes smaller than 0.5 or less negative than -0.5 , and/or regressions that are not significant.

Group data analysis consisted of two analyses. Unpaired $t$ tests were used to determine significant differences between flexor-dominated and non-flexor-dominated fictive scratching episodes for the following parameters: $\mathrm{CP}_{\mathrm{FLEX}}$, flexion phase duration, extension phase duration, flexion-interval phase duration, $r_{\mathrm{FLEX}}, r_{\mathrm{EXT}}, r_{\mathrm{FLEX}-\mathrm{INT}}$, and the percentage time occupied by the flexion, extension, and flexor-interval phase durations relative to their corresponding cycle period. Paired $t$ tests were
1979; Halbertsma, 1983). The same pattern is typically found during fictive locomotion in cats (Frigon and Gossard, 2009), except with electrical stimulation of the mesencephalic locomotor region (MLR) (Yakovenko et al., 2005). During normal scratching in intact cats, however, cycle period seemingly varies with the flexion phase (i.e., flexor-dominated) (Kuhta and Smith, 1990). In the present study, the regulation of cycle period during fictive scratch was evaluated.

In some cats, we succeeded in recording episodes of spontaneous fictive locomotion and pinna-evoked fictive scratch. Figure 1 shows an episode of spontaneous fictive locomotion followed immediately by an episode of fictive scratch. Pinna stimulation stopped fictive locomotion and $\sim 15$ s later, an episode of fictive scratch developed with the typical aiming phase followed 
by vigorous rhythmic alternating activity between flexors and extensors. This episode of fictive locomotion had a cycle period of $1092 \pm 190 \mathrm{~ms}$, an extension phase of $601 \pm 184 \mathrm{~ms}$, and a flexion phase of $379 \pm 74 \mathrm{~ms}$. During fictive scratch, the rhythm was faster with a cycle period of $493 \pm 23 \mathrm{~ms}$, an extension phase of $68 \pm 7$ $\mathrm{ms}$ and a flexion phase of $367 \pm 23 \mathrm{~ms}$. During fictive locomotion the extension and flexion phases respectively occupied $55.0 \%$ and $34.7 \%$ of the cycle, whereas during fictive scratch the extension and flexion phases respectively occupied $13.8 \%$ and $74.4 \%$ of the cycle. The control of cycle period during this episode switched from extensor-dominated during fictive locomotion to flexor-dominated during fictive scratch. Thus, there is a clear difference in the structure of the cycle and in the regulation of cycle period between fictive locomotion and scratch.

A larger sample of episodes (28 episodes in 15 cats) was evaluated to determine how cycle period is regulated during fictive scratching. Figure 2 shows 2 episodes of fictive scratch in 2 cats. In Figure $2 A$, cycle period ( $351 \pm 42 \mathrm{~ms}$ ) changed as a function of the flexion phase, whereas in Figure $2 B$, cycle period $(292 \pm 21 \mathrm{~ms})$ did not change as a function of the duration of the flexion or extension phases, but as a function of the flexion-interval phase duration, or "flexor-interval-dominated."

Across the group ( $n=28$ ), flexor-dominated episodes (20 of 28 episodes; cycle period $=328 \pm 17 \mathrm{~ms}$ ) accounted for the majority of fictive scratching episodes, whereas flexor interval-dominated ( 7 of 28 ; cycle period $=315 \pm 18 \mathrm{~ms}$ ) or invariance ( 1 of 28 ; cycle period $=330 \mathrm{~ms}$ ) accounted for the rest. In the 1 invariant episode $r_{\text {FLEX }}, r_{\text {FLEX-INT }}$, and $r_{\text {EXT }}$ were $0.40,0.45$, and 0.00 , respectively.

Figure 3 compares some parameters of flexor-dominated and "non-flexor-dominated" fictive scratching episodes (unpaired $t$ tests, $p<0.05$ ). There was no significant difference in cycle period and phase durations between flexor-dominated and nonflexor-dominated episodes (Fig. 3A). As expected, $r_{\text {FLEX }}$ was significantly greater in flexor-dominated episodes, whereas $r_{\text {FLEX-INT }}$ was significantly greater during non-flexor-dominated episodes (Fig. $3 B$ ). There was no significant difference in $r_{\text {EXT }}$ between the 2 types of episodes, confirming that the extension phase is not important in determining cycle period during fictive scratching. In flexor-dominated episodes, the flexion phase occupied a significantly greater percentage of the cycle period compared with nonflexor-dominated episodes (Fig. 3C). In non-flexor-dominated episodes, the flexion-interval phase occupied a significantly greater percentage of cycle period compared with flexor-dominated episodes. The extension phase relative to cycle period did not differ in the 2 types of episodes. Thus, although there is a clear flexordominance in the regulation of cycle period during fictive scratch, this bias can change if the percentage of the flexion-interval phase relative to cycle period increases (i.e., if the structure of the rhythm is altered). We previously showed that the extensor-dominance during spontaneous fictive locomotion could be altered if the percentage of
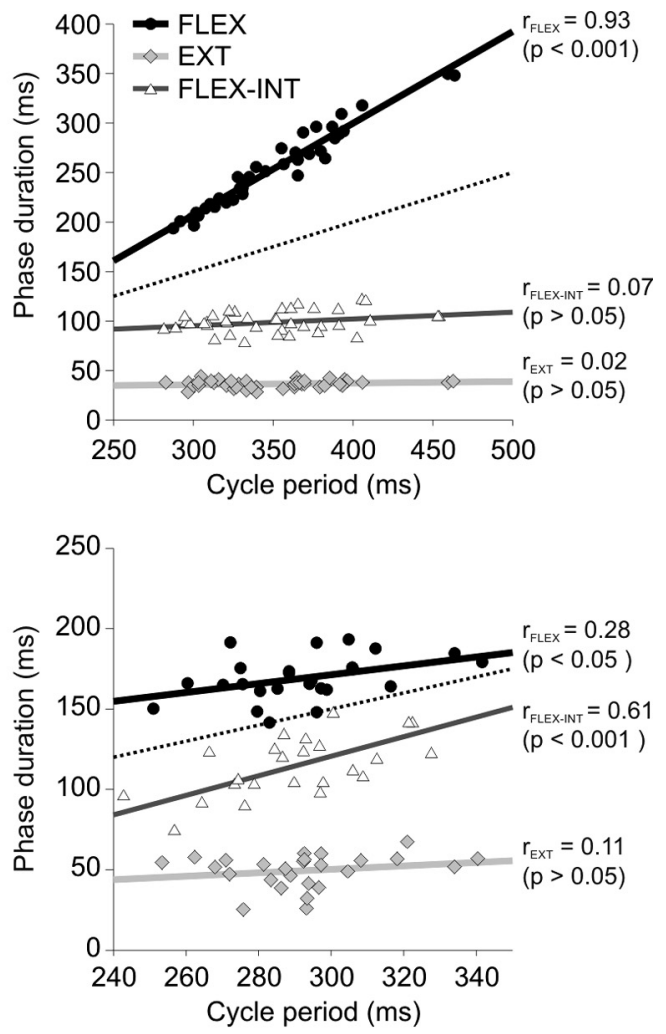

Figure 2. Phase/cycle period relationships during fictive scratching in the decerebrate cat. Left panels show ENG from extensor (MG) and flexor (TA) nerves during flexor-dominated $(\boldsymbol{A})$ and non-flexor-dominated $(\boldsymbol{B})$ fictive scratching episodes in 2 cats. Right (black circles), extension (gray diamonds), and flexion-interval (white triangles) phase durations expressed relative to the corresponding cycle period. The dotted line has a slope of 0.5 and passes through the origin and is used as a reference line.

flexion relative to cycle period increased significantly (Frigon and Gossard, 2009).

Effect of ankle dorsiflexion on the regulation of cycle period during fictive locomotion and scratching

To determine whether sensory inputs from load and/or stretchsensitive receptors influence centrally generated rhythms, slight sustained dorsiflexion of the left ankle, which stretches ankle extensors, was applied during episodes of fictive locomotion and scratch. The effect of ankle dorsiflexion was evaluated during 13 episodes of fictive locomotion in 4 cats and during 11 episodes of fictive scratch in 4 cats. Figure 4 shows the effects of ankle dorsiflexion during 2 fictive locomotor and 2 scratching episodes in 4 cats. It is clear from the raw ENG signals shown in the left panels that both the duration and amplitude of bursts of activity in extensors are greatly increased with dorsiflexion during fictive locomotion (Fig. 4A,B), but not during fictive scratch (Fig. $4 C, D)$. In Figure $4 A$, the spontaneous fictive locomotor episode before dorsiflexion (Ctrl) was extensor-dominated with a cycle period of $1233 \pm 18 \mathrm{~ms}$. During dorsiflexion (DF), this episode remained extensor-dominated but cycle period increased considerably to $1973 \pm 127 \mathrm{~ms}$. In Figure $4 B$, the spontaneous fictive locomotor episode before dorsiflexion was flexor-dominated with a cycle period of $703 \pm 12$ ms. During dorsiflexion, this episode became extensor-dominated as cycle period increased considerably to $1143 \pm 82 \mathrm{~ms}$. In Figure $4 C$ the fictive scratching episode was flexor-dominated before and during dorsiflexion. On the other hand, in the scratching episode depicted in Figure $4 D$, the episode was flexor-dominated before dorsiflexion but 

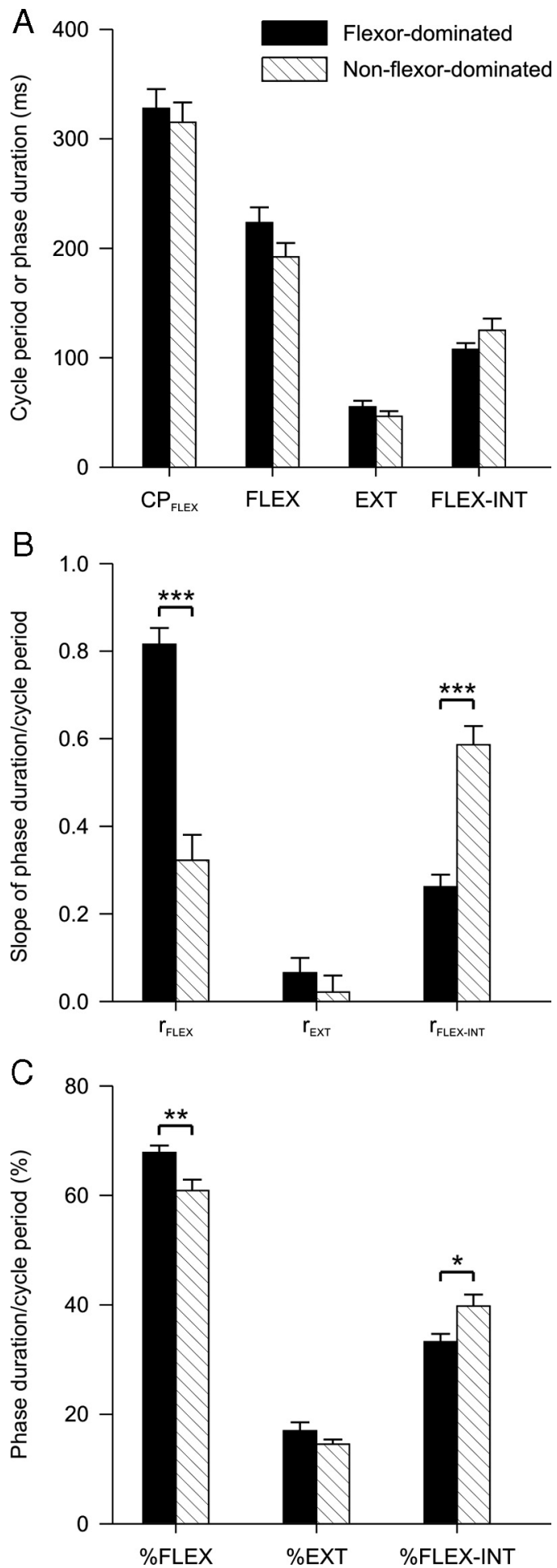

Figure 3. Parameters of the fictive scratching cycle during flexor-dominated and nonflexor-dominated episodes. $\boldsymbol{A}$, Average duration of $\mathrm{CP}_{\mathrm{FLEX}}$ and of the FLEX, EXT, and FLEX-INT phases. $\boldsymbol{B}$, Mean slope of the flexion $\left(r_{\mathrm{FLEX}}\right)$, extension $\left(r_{\mathrm{EXT}}\right)$, and flexion-interval $\left(r_{\mathrm{FLEX}-\mathrm{INT}}\right)$ phase durations/cycle period regressions. $C$, Percentage of flexion, extension, and flexioninterval phase duration as a function of cycle period. Each bar is the mean \pm SE of 20 flexor-dominated and 8 non-flexor-dominated scratching episodes. ${ }^{*} p<0.05$, ${ }^{* *} p<$ $0.01,{ }^{* * *} p<0.01$.

covaried with the extension and flexor-interval phases during dorsiflexion (multiple linear regression analysis $p>0.05$ ).

Figure 5 compares some parameters of spontaneous fictive locomotion and fictive scratching episodes before and during dorsiflexion (paired $t$ tests, $p<0.05$ ). During spontaneous fictive locomotion, ankle dorsiflexion significantly increased cycle period by increasing the duration of the extension phase, while the duration of the flexion phase decreased (Fig. 5A). During fictive scratch, ankle dorsiflexion had no effect on cycle period because the reduction in the flexion phase was counterbalanced by an increase in the extension and/or flexor-interval phases (Fig. 5B). Dorsiflexion reduced $r_{\text {FLEX }}$ during fictive locomotion and scratch while increasing $r_{\mathrm{EXT}}$ (Fig. 5C,D). During spontaneous fictive locomotion, before dorsiflexion, 10 of 13 episodes were extensordominated, 2 of 13 were flexor-dominated, and 1 of 13 was invariant, which is approximately the same repartition previously described (Frigon and Gossard, 2009). However, with dorsiflexion 13 of 13 episodes were extensor-dominated, even those that were flexor-dominated became extensor-dominated (see Fig. $4 B$ for example). During fictive scratch, before dorsiflexion 10 of 11 episodes were flexor-dominated and 1 of 11 was flexion-intervaldominated. During dorsiflexion 7 of 11 remained flexordominated, 1 of 11 was flexion-interval-dominated (not the same as before), 2 of 11 episodes became invariant, and 1 of 11 covaried with the flexion-interval and extension phases (see Fig. $4 D$ for example). Thus, during fictive locomotion, ankle dorsiflexion increases cycle period and strengthens extensor-dominance by increasing the duration of the extension phase. During fictive scratch, however, dorsiflexion does not change cycle period but it weakens flexor-dominance by increasing the duration of the extension and flexion-interval phases.

\section{Effect of nerve stimulation on the regulation of cycle period during fictive locomotion and scratching}

Figures 6 and 7 show the effects of electrically stimulating peripheral nerves during spontaneous fictive locomotion and fictive scratch. More specifically, the effects of stimulating the Pl, LGS and Sart nerves were investigated because inputs from ankle extensors and hip flexors are known to have potent effects on the rhythm generating circuitry during fictive locomotion (Conway et al., 1987; Gossard et al., 1994; Guertin et al., 1995; Perreault et al., 1995; Schomburg et al., 1998; Stecina et al., 2005; Rossignol et al., 2006; Frigon et al., 2010). The locomotor and scratch cycles were subdivided into 4 epochs to determine whether the effects of nerve stimulation on cycle period varied at different time points.

Stimulating the Pl nerve with short trains of 25 pulses at group I afferent strength during spontaneous fictive locomotion reset the rhythm, as previously shown (Conway et al., 1987; Gossard et al., 1994; Frigon et al., 2010), by switching from flexion to extension (Fig. 6A) or by prolonging the ongoing extension phase, therefore delaying the following flexion phase (Fig. 6C). However, during fictive scratching, the same nerve stimulation clearly failed to reset the rhythm because it could not switch the rhythm from flexion to extension (Fig. $6 B$ ), or delay the next flexion phase (Fig. 6D). During spontaneous fictive locomotion, for the group, stimulating the Pl or LGS nerves during EF, MF, and LF significantly reduced $\mathrm{F}$ 1, but had no effect on the duration of the following extension phase (Fig. 6 E). Stimulation during EXT, on the other hand, significantly prolonged the extension phase. The flexion phase (F2) that followed the stimulation was not significantly altered at any epoch. During fictive scratch, for the group, stimulating the Pl or LGS nerves during EF, MF, and LF significantly reduced $\mathrm{F} 1$ and increased the following extension phase. Stimulation during the flexion-interval phase significantly prolonged the FLEX-INT phase (Fig. $6 F$ ). Stimulating during spontaneous fictive locomotion reduced $\mathrm{CP}_{\text {stim }}$ during $\mathrm{EF}, \mathrm{MF}$, and LF, whereas stimulation during the extension phase increased 
$\mathrm{CP}_{\text {stim }}$ (Fig. 6G). The cycle period that followed stimulation was not different from control cycles. During fictive scratch, $\mathrm{CP}_{\text {stim }}$ was significantly decreased with stimulation during MF, increased during LF, and unchanged during EF (Fig. 6H). Stimulation during FLEX-INT significantly increased $\mathrm{CP}_{\text {stim. }}$. The cycle period following the stimulation was reduced during LF only. Thus, although the effects of $\mathrm{Pl}$ stimulation are similar (i.e., an increase of the extension or flexor-interval phases) during fictive locomotion and scratching when the train falls during EXT or FLEX-INT, there is a clear difference with stimulation during the flexion phase. Most notably, stimulation during EF and LF fails to reset the rhythm by eliciting an extension during fictive scratching.

Stimulating the Sart nerve with short trains of 25 pulses at $5 \mathrm{~T}$ (recruiting both group I and II afferents) during spontaneous fictive locomotion primarily reset the rhythm by switching from flexion to extension (Fig. 7A), or slightly prolonged the ongoing extension phase, delaying the following flexion phase (Fig. 7C), as previously shown (Frigon et al., 2010). However, during fictive scratching, the same nerve stimulation during early flexion produced a large prolongation of the flexion phase with a concomitant increase in cycle period (Fig. $7 B$ ). Stimulation during the flexion-interval phase had no effect on the onset of the following flexor burst but prolonged its duration (Fig. 7D). During spontaneous fictive locomotion, for the group, stimulating the Sart nerve during $\mathrm{EF}, \mathrm{MF}$, and LF significantly reduced F1, but had no effect on the duration of the following extension phase (Fig. 7E). Stimulation during EXT, on the other hand, significantly prolonged the extension phase. The flexion phase that followed the stimulation was not significantly altered at any epoch. During fictive scratching, for the group, stimulating the Sart nerve significantly prolonged $\mathrm{F} 1$ during $\mathrm{EF}$, but reduced $\mathrm{F} 1$ during $\mathrm{MF}$ (Fig. $7 F$ ). The flexion-interval phase that followed was reduced during LF. Stimulation during FLEX-INT prolonged the flexion-interval phase. F2 was significantly increased during MF, LF, and FLEX-INT. During spontaneous fictive locomotion, for the group, stimulating the Sart nerve during EF, MF, or LF significantly reduced $\mathrm{CP}_{\text {stim }}$ relative to control cycles, whereas stimulation during EXT slightly increased $\mathrm{CP}_{\text {stim }}$ (Fig. $7 G)$. The cycle period that followed stimulation was not different from control cycles. During fictive scratching, Sart nerve stimulation considerably increased $\mathrm{CP}_{\text {stim }}$

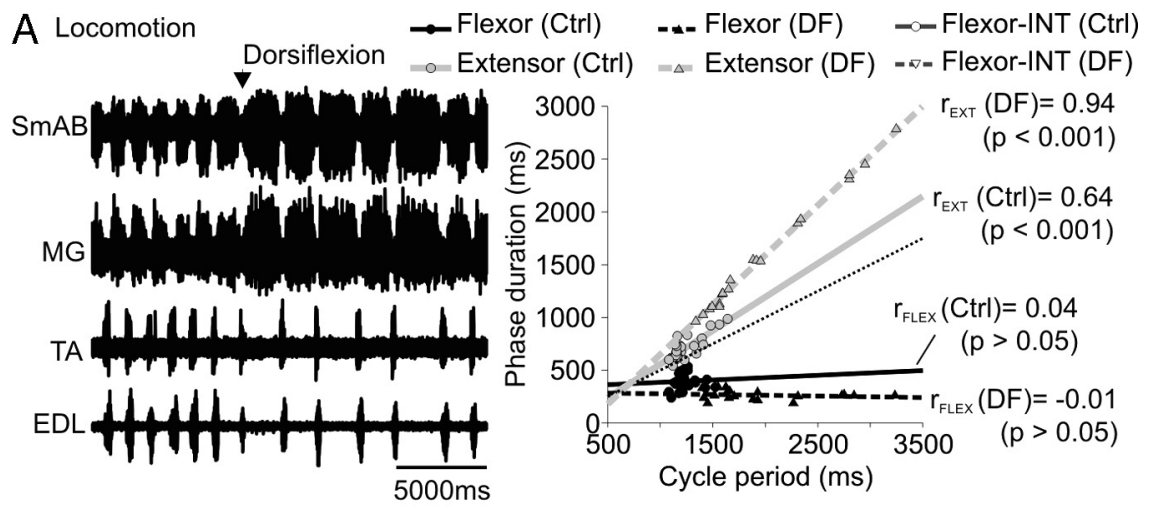

B Locomotion
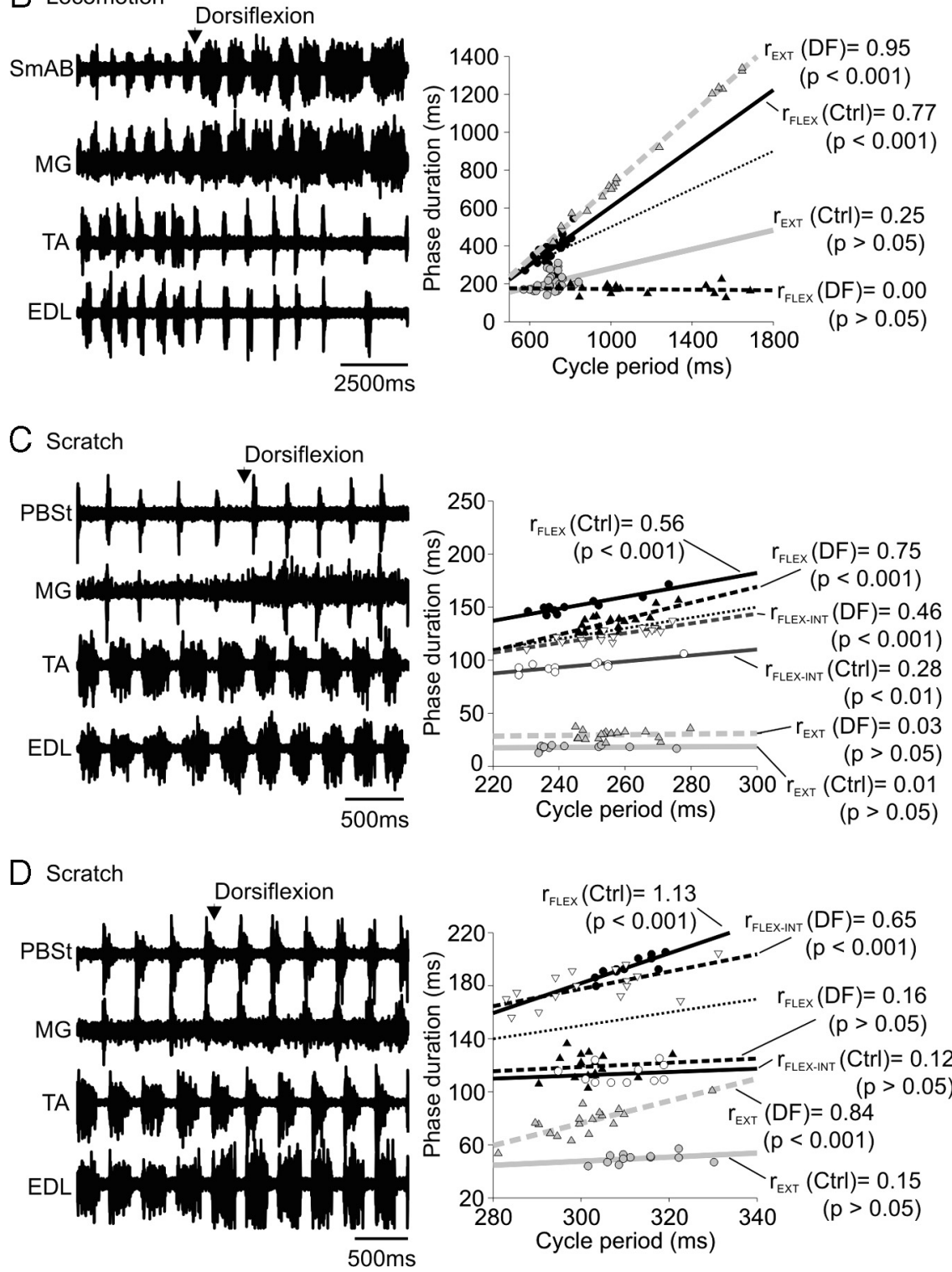

Figure 4. Effects of ankle dorsiflexion during spontaneous fictive locomotion and fictive scratching. The left panels show ENG from extensor (SmAB and MG during fictive locomotion and PBSt and MG during fictive scratching) and flexor (TA and EDL) nerves during extensor-dominated $(\boldsymbol{A})$ and flexor-dominated $(\boldsymbol{B})$ locomotor episodes in 2 cats, and during 2 flexor-dominated $(\boldsymbol{C}, \boldsymbol{D})$ scratching episodes in 2 cats. Right panels show scatter plots and regression lines for extension (gray) and flexion (black) phase durations relative to cycle period before (Ctrl) and during (DF) dorsiflexion. During fictive scratching, the flexion-phase interval (white) duration as a function of cycle period is also represented. The dotted line has a slope of 0.5 and passes through the origin and is used as a reference line. 

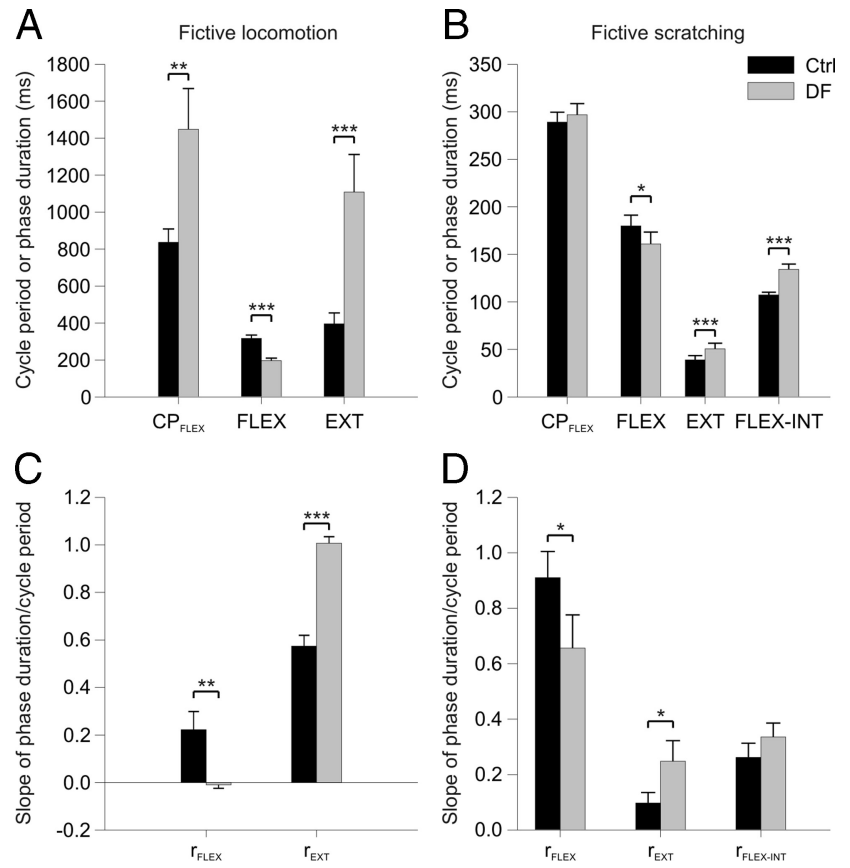

Figure 5. Effects of ankle dorsiflexion during spontaneous fictive locomotion and fictive scratching for the group. $\boldsymbol{A}, \boldsymbol{B}$, Average duration of the $\mathrm{CP}_{\mathrm{FLEX}}$, and of the FLEX, EXT, and FLEX-INT phases before (Ctrl) and during (DF) dorsiflexion. $C, D$, Mean slope of the flexion $\left(r_{\text {FLEX }}\right)$, exten$\operatorname{sion}\left(r_{\text {EXT }}\right)$, and flexion-interval $\left(r_{\text {FLEX-INT }}\right)$ phase durations/cycle period regressions before and during dorsiflexion. Each bar is the mean \pm SE of 20 flexor-dominated and 8 non-flexordominated scratching episodes. ${ }^{*} p<0.05,{ }^{* *} p<0.01,{ }^{* * *} p<0.01$.

during $\mathrm{EF}$ and reduced $\mathrm{CP}_{\text {stim }}$ during mid-flexion (Fig. $7 \mathrm{H}$ ). The cycle period that followed was increased with stimulation during MF, LF and FLEX-INT, which was due to an increase in F2. Because cycle period is much shorter during fictive scratching, compared with fictive locomotion, the train continued into the next flexion phase, which likely explains the last result. Inputs from the Sart nerve appear to mostly influence the scratch rhythm-generating circuitry during early flexion, and this outcome is opposite to what is observed during spontaneous fictive locomotion.

\section{Discussion}

In the present study, cycle period during fictive scratching was adjusted by predominantly varying flexion phase duration (i.e., flexor-dominated), contrary to spontaneous fictive locomotion, which is primarily extensor-dominated (Frigon and Gossard, 2009). Moreover, sensory inputs from load- and/or stretchsensitive receptors from ankle extensors and from sartorius exerted markedly different effects on cycle period, phase durations, and phase transitions during fictive locomotion and scratch, providing evidence that locomotion and scratch are produced by specialized rhythm-generating mechanisms.

\section{Evidence for specialized rhythm-generating mechanisms}

Cycle period can be regulated by changing the duration of the extension phase, the flexion phase, or both. During fictive locomotion occurring spontaneously, or in cats with a spinal transection, cycle period varied more with extension phase duration (i.e., extensor-dominated) (Frigon and Gossard, 2009); which closely resembles intact walking where cycle period varies with stance duration (Goslow et al., 1973; Grillner et al., 1979; Halbertsma, 1983; Grillner and Dubuc, 1988). Conversely, cycle period during fictive scratch varied with flexion phase duration
(Figs. 1, 2). A flexor-dominated pattern is also found during normal scratch (Kuhta and Smith, 1990).

However, it should be noted that cycle period is primarily flexor-dominated during fictive locomotion evoked by electrically stimulating the MLR, a rhythm where the flexion phase generally occupies $>50 \%$ of the cycle (Yakovenko et al., 2005). On the other hand, during spontaneous fictive locomotion, cycle period is primarily extensor-dominated and the extension phase is usually $>50 \%$ of the cycle (Frigon and Gossard, 2009). Because MLR stimulation is usually performed following a precollicular/ postmammillary section while spontaneous fictive locomotion occurs following a precollicular/premammillary section without electrical stimulation, different descending drives on a set of coupled symmetric oscillators could account for the different patterns of dominance (Yakovenko et al., 2005). However, in some locomotor episodes, cycle period was determined by variations in the shorter period (Frigon and Gossard, 2009). Moreover, during fictive locomotion in complete spinal cats (acute or chronic) the rhythm is always extensor-dominated (Frigon and Gossard, 2009); thus the pattern of dominance cannot be solely attributed to different descending drives.

That locomotion and scratch are produced by a single rhythm generator was largely based on 3 observations (Deliagina et al., 1975, 1981; Baev, 1978; Berkinblit et al., 1978b; Gelfand et al., 1988; Perreault et al., 1999). First, rhythmically active neurons for locomotion and scratch can be colocalized at the same spinal segments (Gelfand et al., 1988), and segments L3-L4 in the cat appear to be critical for generating locomotion (Marcoux and Rossignol, 2000; Langlet et al., 2005) and scratch (Berkinblit et al., 1978a). However, rhythmogenic activity is distributed over several spinal segments during locomotion (Cowley and Schmidt, 1997; Kiehn, 2006) and scratch (Deliagina et al., 1983) and, consequently, it is not surprising to find rhythmically active neurons at the same spinal segments in both movements. Moreover, although some interneurons can be active during scratch and locomotion, some interneurons are specialized for one task (Berkowitz, 2007, 2008). In other words, some neurons active in one task are silent in another. Thus, colocalization of rhythmically active neurons during locomotion and scratch does not indicate that both rhythms are produced by the same configuration of the rhythm-generating circuitry, only that some neuronal elements are shared to produce multiple movements.

The second observation was that muscle groups or interneurons active in flexion or extension during locomotion remained active in the same phase during scratching (Deliagina et al., 1975, 1981). However, there are several exceptions. For example, the PBSt is most often coactive with flexors such as TA and EDL during fictive locomotion, whereas during scratch it discharges with extensors, such as SmAB, LGS, and MG (Fig. 2) (Deliagina et al., 1975, 1981; Berkinblit et al., 1980; Degtyarenko et al., 1998; Lafreniere-Roula and McCrea, 2005). Other examples include flexor digitorum longus (Degtyarenko et al., 1998) and peroneus longus (Lafreniere-Roula and McCrea, 2005; Stecina et al., 2005), which are grouped into different synergies during locomotion and scratch. Although it cannot be excluded that the same rhythm generator produces a flexor command in locomotion and an extensor command during scratch, it is easier to conceptualize different rhythm-generating mechanisms in the two behaviors. Moreover, although some spinal interneurons are phase-locked during scratch and locomotion (Berkinblit et al., 1978a; Baev and Kostyuk, 1981; Gelfand et al., 1988), others have their peak discharge rate during different phases (Berkinblit et al., 1978a,b; Berkowitz, 2008). 
The third observation was that sensory inputs exerted similar effects during scratch and locomotion (Perreault et al., 1999). For example, stimulating the $\mathrm{Pl}$ nerve during the flexion phase of fictive scratching reduced flexor burst duration and advanced extensor burst onset (Perreault et al., 1999). Although this was taken as evidence for resetting of the scratch rhythm, extensor burst onset was not immediately triggered by the stimulation (see Perreault et al., 1999, their Fig. 6). In Perreault et al. (1999), while stimulation during extension slightly increased extensor burst duration, cycle period was rarely altered, as in the present study (Fig. 6). This is strikingly different from what is seen during spontaneous fictive locomotion where Pl nerve stimulation during flexion terminates flexion and rapidly initiates extension, whereas stimulation during extension prolongs the extension phase and cycle period concurrently (Fig. $6)$. One possible explanation for discrepancies in the comparison between fictive locomotion and scratch is that the fictive locomotion was induced by MLR stimulation in Perreault et al. (1999) while it occurred spontaneously in our study (see Frigon and Gossard, 2009). Differences in the actions of hip or ankle muscle afferent stimulation during MLR-evoked and spontaneous fictive locomotion are discussed in detail in Frigon et al. (2010).

Furthermore, other observations from the present study argue for some distinction in control mechanisms at the rhythm-generation level during fictive locomotion and scratch. For example, sustained stimulation of stretch-sensitive ankle extensor afferents by dorsiflexing the ankle considerably increased cycle period by prolonging the extension phase during fictive locomotion, but during fictive scratching the slight increase in extension and flexion-interval phases was counterbalanced by reducing the flexion phase, leaving cycle period unchanged (Figs. 4, 5). In addition, during fictive locomotion, stimulating the Sart nerve at $5 \mathrm{~T}$ in early or mid-flexion terminated flexion and reset the rhythm to extension (Fig. 7). During fictive scratch, however, the same stimulation during early flexion prolonged cycle period and flexion phase concomitantly (Fig. 7). Therefore, ankle extensor or hip flexor inputs, which have potent effects during fictive locomotion, have weaker or even opposite effects during fictive scratching.

Stimulating cutaneous or muscle nerves also evoked different patterns and modulation of postsynaptic potentials (PSPs) during fictive locomotion and scratch (Degtyarenko et al., 1998). For instance, PSPs evoked in hindlimb motoneurons by stimulating
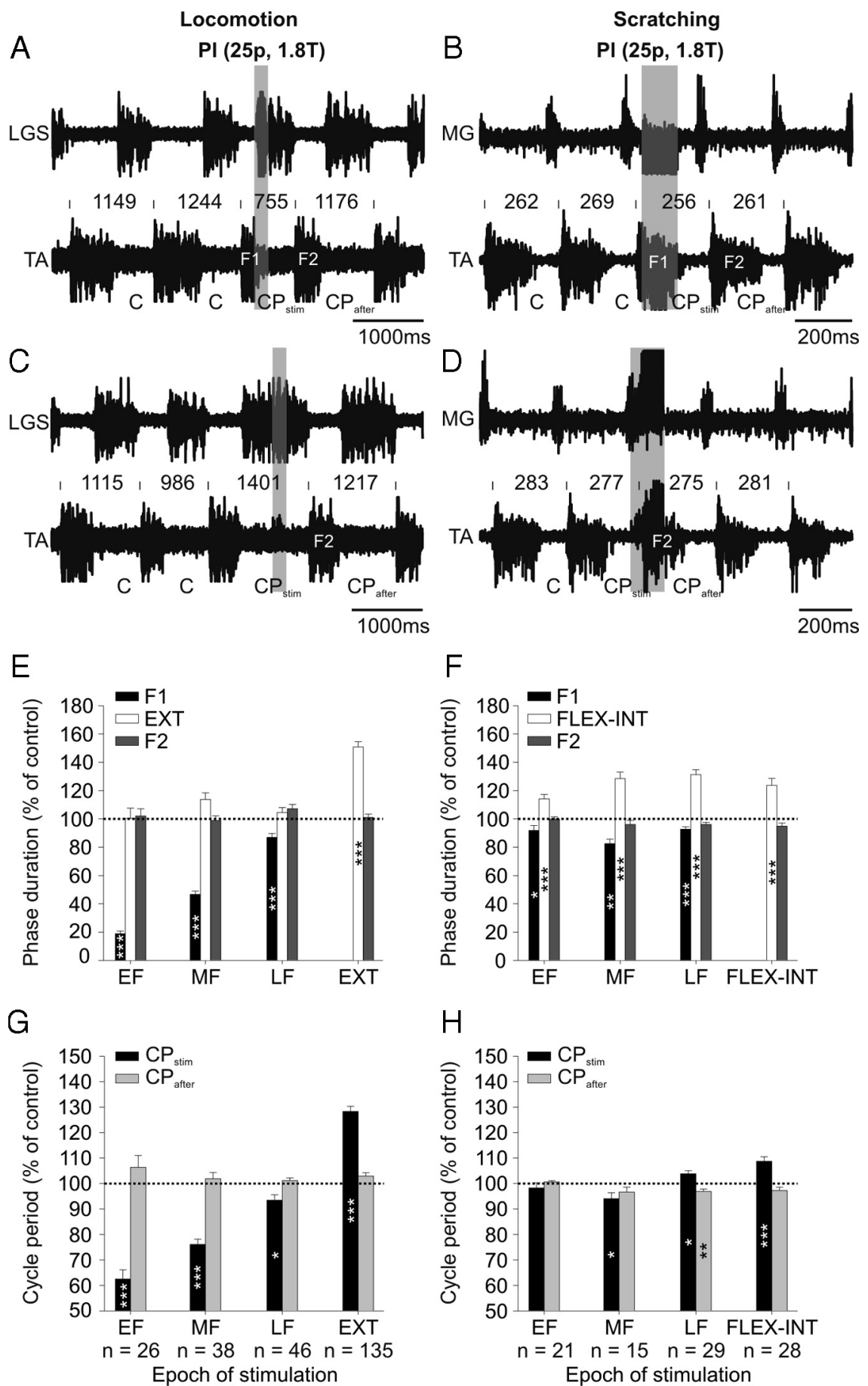

Figure 6. Effects of PI or LGS nerve stimulation during fictive locomotion and scratching. $\boldsymbol{A}-\boldsymbol{D}$, Top panels show ENG from extensor (LGS or MG) and flexor (TA) nerves in 2 cats with short trains of electrical stimuli to the PI nerve (25p at 1.8T, 200 Hz) during early flexion in fictive locomotion $(\boldsymbol{A})$ and scratching $(\boldsymbol{B})$ and during the late extension $(\boldsymbol{C})$ or late flexion $(\boldsymbol{D})$ interval epochs of fictive locomotion and scratching, respectively. The onset of the flexion phase and cycle period is indicated above the TA waveform. $\boldsymbol{E}, \boldsymbol{F}$, Average phase duration of the flexion phase F1 or F2 stimulation during EF, MF, LF, and EXT or FLEX-INT epochs, expressed relative to control flexion phase durations. The duration of the extension or flexion-interval phase (i.e., between F1 and F2) is also shown. Note that when the stimuli fall in EXT or FLEX-INT the extension or flexion-interval phase is the stimulated phase. $\mathbf{G}, \boldsymbol{H}$, Mean flexor cycle period $\mathrm{CP}_{\text {stim }}$ and $\mathrm{CP}_{\text {after }}$ stimulation measured for the same epochs as in $\boldsymbol{E}, \boldsymbol{F}$. The number of data points used in each condition to calculate phase durations and cycle periods is indicated below the epoch of $\mathbf{G}, \boldsymbol{H}$. Each bar is the mean $\pm \mathrm{SE}$. Asterisks indicate significant differences with control cycles (paired $t$ tests). ${ }^{*} p<0.05,{ }^{* *} p<0.01,{ }^{* * *} p<0.01$.

cutaneous nerves were prominent during fictive locomotion and showed strong phasic modulation, whereas during fictive scratching, PSPs were suppressed with little phase modulation. On the other hand, PSPs evoked by muscle nerve stimulation 

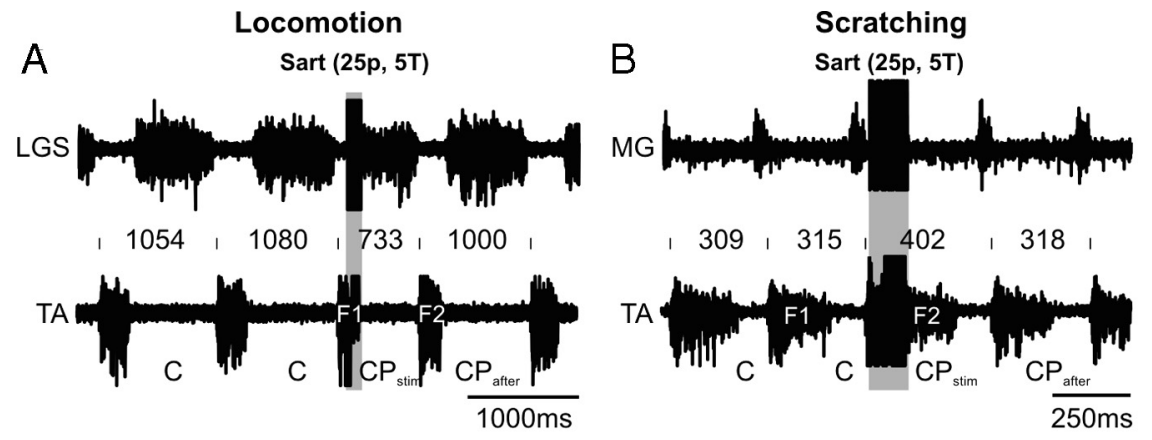

C
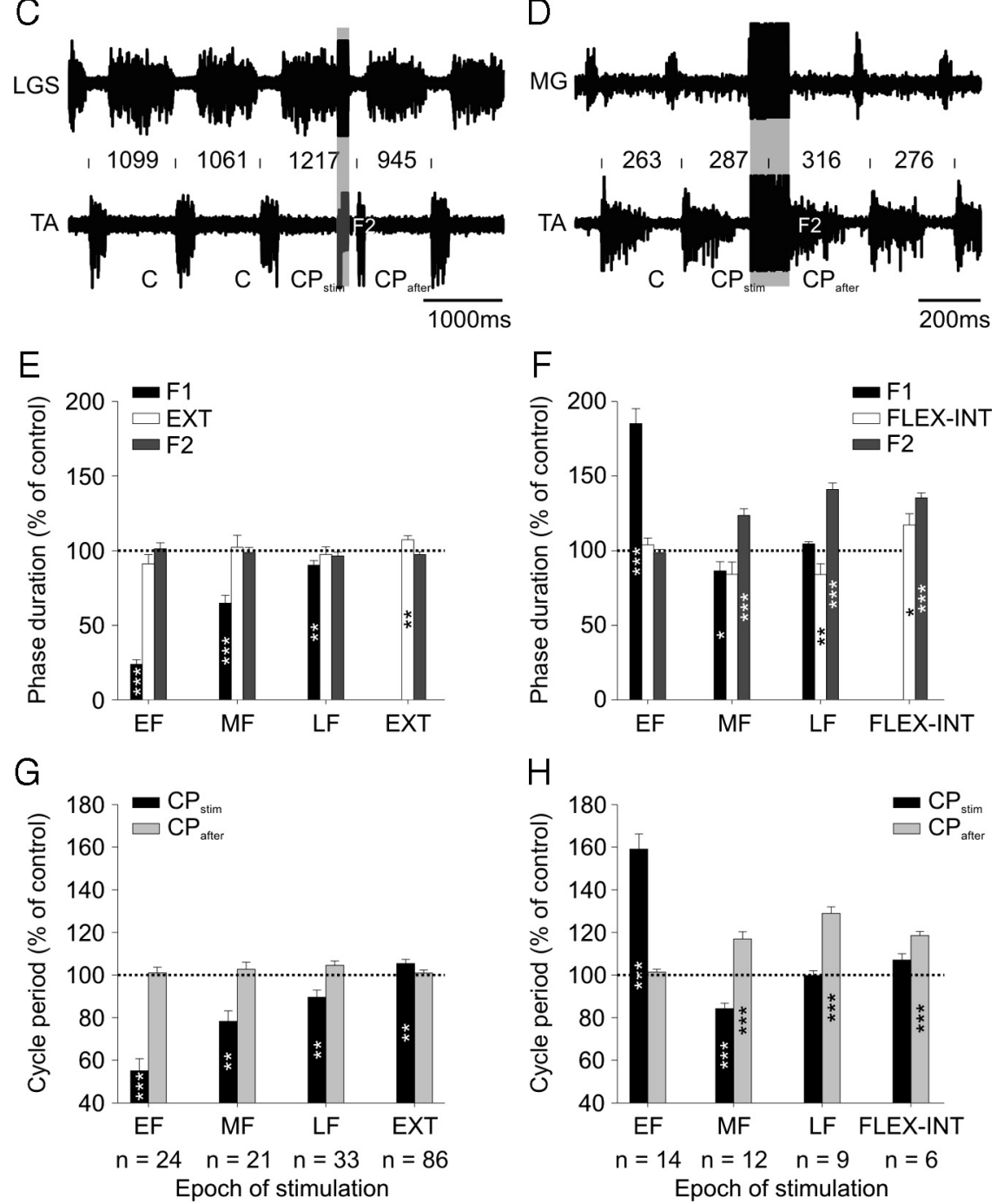

Figure 7. Effects of Sart nerve stimulation during fictive locomotion and scratching. $A-D$, Top panels show ENG from extensor (LGS or MG) and flexor (TA) nerves in 2 cats with short trains of electrical stimuli to the Sart nerve (25p at $5 \mathrm{~T}, 200 \mathrm{~Hz}$ ) during early flexion in fictive locomotion $(\boldsymbol{A})$ and scratching $(\boldsymbol{B})$ and during the late extension $(\boldsymbol{C})$ or late flexion $(\boldsymbol{D})$ interval epochs of fictive locomotion and scratching, respectively. The onset of the flexion phase and cycle period is indicated above the TA waveform. $\boldsymbol{E}, \boldsymbol{F}$, Average phase duration of the flexion phase F1 or F2 stimulation during EF, MF, LF, and EXT or FLEX-INT epochs, expressed relative to control flexion phase durations. The duration of the extension or flexion-interval phase (i.e., between F1 and F2) is also shown. Note that when the stimuli fall in EXT or FLEX-INT the extension or flexion-interval phase is the stimulated phase. $\boldsymbol{G}, \boldsymbol{H}$, Mean flexor cycle period $\mathrm{CP}_{\text {stim }}$ and $\mathrm{CP}_{\text {after }}$ stimulation measured for the same epochs as in $\boldsymbol{E}, \boldsymbol{F}$. The number of data points used in each condition to calculate phase durations and cycle periods is indicated below the epoch of $\boldsymbol{G}, \boldsymbol{H}$. Each bar is the mean $\pm \mathrm{SE}$. Asterisks indicate significant differences with control cycles (paired $t$ tests). ${ }^{*} p<0.05,{ }^{* *} p<0.01,{ }^{* *} p<0.01$.

were strongly modulated during fictive locomotion and scratch but the modulation pattern was often reversed. Stimulating the MLR also produced short-latency PSPs in hindlimb motoneurons, which were strongly modulated during fictive locomotion
(Shefchyk and Jordan, 1985). However, during fictive scratch these PSPs were abolished (Degtyarenko et al., 1998). Thus, processing of peripheral and supraspinal inputs is strikingly different during fictive locomotion and scratch, which is also exemplified by differences in presynaptic inhibition (Côté and Gossard, 2003).

The level of presynaptic inhibition of primary afferents (and number of antidromic discharges) is greatly decreased during fictive scratch compared with fictive locomotion, which is inconsistent with weak effects from some sensory inputs during fictive scratch (Figs. 4-6). If inputs from primary afferents are not gated out by presynaptic inhibition during scratching, then it must be occurring further down in spinal pathways. Differences in accessing the rhythm generator during fictive locomotion and scratch are most easily explained by different sensory gating at the interneuronal level. Our data does not permit determining the level of the CPG where the differential integration and processing of sensory inputs during locomotion and scratch occur. However, because such inputs can deeply modify (and entrain) phase transitions and durations, it is recognized that they must access the rhythm-generating circuitry (Kriellaars et al., 1994; Hultborn et al., 1998; Pearson et al., 1998; Rossignol et al., 2006). Interneurons gating sensory feedback need not be part of the rhythmgenerating circuitry but they have to be controlled by it.

\section{Reconfiguration of the}

rhythm-generating circuitry

Specific configurations of the rhythmgenerating interneuronal circuitry might be required to produce unique features of locomotion and scratching. For instance, during normal walking or "speed-matched" spontaneous fictive locomotion the extension phase occupies more of the cycle than the flexion phase (Frigon and Gossard, 2009). In contrast, during normal or fictive scratch the extension phase is very brief (Deliagina et al., 1975, 1981). The descending commands required to initiate fictive locomotion and scratch also differ. Locomotion is initiated by supraspinal structures located in the mesencephalon (Jordan et al., 2008), whereas scratching is seemingly mediated by propriospinal neurons (Sherrington and Laslett, 1903; Sherrington, 1906). Certain drugs normally released by pathways originating in the brainstem also change the rhythm from "locomotor-like" to "scratch-like" in neonatal rats (Kiehn and Kjaerulff, 1996). For example, with 
serotonin, the rhythm is fast and PBSt is coactive with extensors (i.e., scratch-like), whereas with dopamine the rhythm is slow with PBSt coactive with flexors (i.e., locomotor-like). Thus, different descending drives or modulatory inputs can produce specific and distinct configurations of the spinal rhythm-generating network, which could mediate differences in control mechanisms from one task to another.

Although Orlovsky and collaborators argued for the same central generator for fictive locomotion and scratch, their model showed that input-output connections to and from locomotor and scratch rhythm generators differed (Berkinblit et al., 1978b), which is most easily accomplished by reconfiguring the rhythmgenerating network, or parts of the circuitry. Work in several vertebrate species (Berkowitz, 2007, 2008; Li et al., 2007; McLean et al., 2007, 2008; Liao and Fetcho, 2008) clearly established that different rhythmic movements are generated by shared and specialized circuitry. Activating and silencing some neurons from one task to another would reconfigure the network. Although this probably occurs continuously to some extent during a given behavior (McLean et al., 2008), shifting the control of cycle period, phase durations, and phase transitions from locomotion to scratching indicates that both rhythms have very specialized control mechanisms, which could be due to distinct configurations of the rhythm-generating circuitry.

\section{References}

Baev KV, Kostyuk PG (1981) Primary afferent depolarization evoked by the activity of spinal scratching generator. Neuroscience 6:205-215.

Baev KV, Esipenko VB, Shimansky YP (1991) Afferent control of central pattern generators: experimental analysis of scratching in the decerebrate cat. Neuroscience 40:239-256.

Bayev KV (1978) Central locomotor program for the cat's hindlimb. Neuroscience 3:1081-1092.

Berkinblit MB, Deliagina TG, Feldman AG, Gelfand IM, Orlovsky GN (1978a) Generation of scratching. 1. Activity of spinal interneurons during scratching. J Neurophysiol 41:1040-1057.

Berkinblit MB, Deliagina TG, Feldman AG, Gelfand IM, Orlovsky GN (1978b) Generation of scratching. II. Nonregular regimes of generation. J Neurophysiol 41:1058-1069.

Berkinblit MB, Deliagina TG, Orlovsky GN, Feldman AG (1980) Activity of motoneurons during fictitious scratch reflex in the cat. Brain Res 193:427-438.

Berkowitz A (2007) Spinal interneurons that are selectively activated during fictive flexion reflex. J Neurosci 27:4634-4641.

Berkowitz A (2008) Physiology and morphology of shared and specialized spinal interneurons for locomotion and scratching. J Neurophysiol 99:2887-2901.

Conway BA, Hultborn H, Kiehn O (1987) Proprioceptive input resets central locomotor rhythm in the spinal cat. Exp Brain Res 68:643-656.

Côté MP, Gossard JP (2003) Task-dependent presynaptic inhibition. J Neurosci 23:1886-1893.

Cowley KC, Schmidt BJ (1997) Regional distribution of the locomotor pattern-generating network in the neonatal rat spinal cord. J Neurophysiol 77:247-259.

Degtyarenko AM, Simon ES, Norden-Krichmar T, Burke RE (1998) Modulation of oligosynaptic cutaneous and muscle afferent reflex pathways during fictive locomotion and scratching in the cat. J Neurophysiol 79:447-463.

Delcomyn F (1980) Neural basis of rhythmic behavior in animals. Science 210:492-498.

Deliagina TG, Feldman AG, Gelfand IM, Orlovsky GN (1975) On the role of central program and afferent inflow in the control of scratching movements in the cat. Brain Res 100:297-313.

Deliagina TG, Orlovsky GN, Perret C (1981) Efferent activity during fictitious scratch reflex in the cat. J Neurophysiol 45:595-604.

Deliagina TG, Orlovsky GN, Pavlova GA (1983) The capacity for generation of rhythmic oscillations is distributed in the lumbosacral spinal cord of the cat. Exp Brain Res 53:81-90.
Feldberg W, Fleischhauer K (1960) Scratching movements evoked by drugs applied to the upper cervical cord. J Physiol 151:502-517.

Frigon A (2009) Reconfiguration of the spinal interneuronal network during locomotion in vertebrates. J Neurophysiol 101:2201-2203.

Frigon A, Gossard JP (2009) Asymmetric control of cycle period by the spinal locomotor rhythm generator in the adult cat. J Physiol 587: 4617-4628.

Frigon A, Sirois J, Gossard JP (2010) The effects of ankle and hip muscle afferent inputs on rhythm generation during fictive locomotion. J Neurophysiol 103:1597-1605.

Gelfand IM, Orlovsky GN, Shik ML (1988) Locomotion and scratching in tetrapods. In: Neural control of rhythmic movements in vertebrates (Cohen AH, Rossignol S, Grillner S, eds), pp 167-199. New York: Wiley.

Goslow GE Jr, Reinking RM, Stuart DG (1973) The cat step cycle: hind limb joint angles and muscle lengths during unrestrained locomotion. J Morphol 141:1-41.

Gossard JP, Brownstone RM, Barajon I, Hultborn H (1994) Transmission in a locomotor-related group Ib pathway from hindlimb extensor muscles in the cat. Exp Brain Res 98:213-228.

Grillner S (1981) Control of locomotion in bipeds, tetrapods, and fish. In: Handbook of physiology. Sec 1: The nervous system, Vol II (Brookhart JM, Mountcastle VB, eds), pp 1179-1236. Bethesda, MD: American Physiological Society.

Grillner S, Dubuc R (1988) Control of locomotion in vertebrates: spinal and supraspinal mechanisms. In: Functional recovery in neurological disease (Waxman SG, ed), pp 425-453. New York: Raven.

Grillner S, Halbertsma J, Nilsson J, Thorstensson A (1979) The adaptation to speed in human locomotion. Brain Res 165:177-182.

Guertin P, Angel MJ, Perreault MC, McCrea DA (1995) Ankle extensor group I afferents excite extensors throughout the hindlimb during fictive locomotion in the cat. J Physiol 487:197-209.

Halbertsma JM (1983) The stride cycle of the cat: the modelling of locomotion by computerized analysis of automatic recordings. Acta Physiol Scand Suppl 521:S1-S75.

Hultborn H, Conway B, Gossard JP, Brownstone R, Fedirchuk B, Schomburg ED (1998) How do we approach the locomotor network in the mammalian spinal cord? Ann N Y Acad Sci 860:70-82.

Jordan LM, Liu J, Hedlund PB, Akay T, Pearson KG (2008) Descending command systems for the initiation of locomotion in mammals. Brain Res Rev 57:183-191.

Kiehn O (2006) Locomotor circuits in the mammalian spinal cord. Annu Rev Neurosci 29:279-306.

Kiehn O, Kjaerulff O (1996) Spatiotemporal characteristics of 5-HT and dopamine-induced rhythmic hindlimb activity in the in vitro neonatal rat. J Neurophysiol 75:1472-1482.

Kriellaars DJ, Brownstone RM, Noga BR, Jordan LM (1994) Mechanical entrainment of fictive locomotion in the decerebrate cat. J Neurophysiol 71:2074-2086.

Kuhta PC, Smith JL (1990) Scratch responses in normal cats: hindlimb kinematics and muscle synergies. J Neurophysiol 64:1653-1667.

Lafreniere-Roula M, McCrea DA (2005) Deletions of rhythmic motoneuron activity during fictive locomotion and scratch provide clues to the organization of the mammalian central pattern generator. J Neurophysiol 94:1120-1132.

Langlet C, Leblond H, Rossignol S (2005) The mid-lumbar segments are needed for the expression of locomotion in chronic spinal cats. J Neurophysiol 93:2474-2488.

Li WC, Sautois B, Roberts A, Soffe SR (2007) Reconfiguration of a vertebrate motor network: specific neuron recruitment and contextdependent synaptic plasticity. J Neurosci 27:12267-12276.

Liao JC, Fetcho JR (2008) Shared versus specialized glycinergic spinal interneurons in axial motor circuits of larval zebrafish. J Neurosci 28: 12982-12992.

Marcoux J, Rossignol S (2000) Initiating or blocking locomotion in spinal cats by applying noradrenergic drugs to restricted lumbar spinal segments. J Neurosci 20:8577-8585.

McCrea DA (1998) Neuronal basis of afferent-evoked enhancement of locomotor activity. Ann N Y Acad Sci 860:216-225.

McCrea DA, Rybak IA (2008) Organization of mammalian locomotor rhythm and pattern generation. Brain Res Rev 57:134-146.

McLean DL, Fan J, Higashijima S, Hale ME, Fetcho JR (2007) A topographic map of recruitment in spinal cord. Nature 446:71-75. 
McLean DL, Masino MA, Koh IY, Lindquist WB, Fetcho JR (2008) Continuous shifts in the active set of spinal interneurons during changes in locomotor speed. Nat Neurosci 11:1419-1429.

Pearson KG, Rossignol S (1991) Fictive motor patterns in chronic spinal cats. J Neurophysiol 66:1874-1887.

Pearson KG, Misiaszek JE, Fouad K (1998) Enhancement and resetting of locomotor activity by muscle afferents. Ann N Y Acad Sci 860:203-215.

Perreault MC, Angel MJ, Guertin P, McCrea DA (1995) Effects of stimulation of hindlimb flexor group II afferents during fictive locomotion in the cat. J Physiol 487:211-220.

Perreault MC, Enriquez-Denton M, Hultborn H (1999) Proprioceptive control of extensor activity during fictive scratching and weight support compared to fictive locomotion. J Neurosci 19:10966-10976.

Rossignol S (1996) Neural control of stereotypic limb movements. In: Handbook of physiology. Sec 12: Exercise: regulation and integration of multiple systems (Rowell LB, Sheperd JT, eds), pp 173-216. New York: Oxford UP.

Rossignol S, Dubuc R, Gossard JP (2006) Dynamic sensorimotor interactions in locomotion. Physiol Rev 86:89-154.
Schomburg ED, Petersen N, Barajon I, Hultborn H (1998) Flexor reflex afferents reset the step cycle during fictive locomotion in the cat. Exp Brain Res 122:339-350.

Shefchyk SJ, Jordan LM (1985) Excitatory and inhibitory post-synaptic potentials in alpha-motoneurons produced during fictive locomotion by stimulation of the mesencephalic locomotor region. J Neurophysiol 53:1345-1355.

Sherrington CS (1906) The integrative action of the nervous system. New Haven, CT: Yale UP.

Sherrington CS, Laslett EE (1903) Observations on some spinal reflexes and the interconnection of spinal segments. J Physiol 29:58-96.

Stecina K, Quevedo J, McCrea DA (2005) Parallel reflex pathways from flexor muscle afferents evoking resetting and flexion enhancement during fictive locomotion and scratch in the cat. J Physiol 569:275-290.

Stein PS (2005) Neuronal control of turtle hindlimb motor rhythms. J Comp Physiol A Neuroethol Sens Neural Behav Physiol Neuroethol Sens Neural Behav Physiol 191:213-229.

Yakovenko S, McCrea DA, Stecina K, Prochazka A (2005) Control of locomotor cycle durations. J Neurophysiol 94:1057-1065. 